\title{
Article \\ Effect of Fermented Rice Drink "Amazake" on Patients with Nonalcoholic Fatty Liver Disease and Periodontal Disease: A Pilot Study
}

\author{
Yumiko Nagao ${ }^{1,2, *(\mathbb{D}, \text { Hirokazu Takahashi }}{ }^{2,3}$, Atsushi Kawaguchi ${ }^{4}$ and Hiroshi Kitagaki ${ }^{5}$ (D) \\ 1 Department of Public Health, Graduate School of Medicine, Juntendo University, Bunkyo-ku, \\ Tokyo 113-8421, Japan \\ 2 Liver Center, Saga University Hospital, Nabeshima, Saga 849-8501, Japan; takahas2@cc.saga-u.ac.jp \\ 3 Division of Metabolism and Endocrinology, Faculty of Medicine, Saga University, Nabeshima, \\ Saga 849-8501, Japan \\ 4 Education and Research Center for Community Medicine, Faculty of Medicine, Saga University, Nabeshima, \\ Saga 849-8501, Japan; akawa@cc.saga-u.ac.jp \\ 5 Faculty of Agriculture, Saga University, Honjo-cho, Saga 840-8502, Japan; ktgkhrs@cc.saga-u.ac.jp \\ * Correspondence: y.nagao.qd@juntendo.ac.jp; Tel.: +81-03-5802-1049
}

Citation: Nagao, Y.; Takahashi, H.; Kawaguchi, A.; Kitagaki, H. Effect of Fermented Rice Drink "Amazake" on Patients with Nonalcoholic Fatty Liver Disease and Periodontal Disease: A Pilot Study. Reports 2021, 4, 36. https://doi.org/10.3390/ reports 4040036

Academic Editor: Elisabetta Albi

Received: 18 September 2021

Accepted: 8 October 2021

Published: 13 October 2021

Publisher's Note: MDPI stays neutral with regard to jurisdictional claims in published maps and institutional affiliations.

Copyright: (c) 2021 by the authors. Licensee MDPI, Basel, Switzerland. This article is an open access article distributed under the terms and conditions of the Creative Commons Attribution (CC BY) license (https:/ / creativecommons.org/licenses/by/ $4.0 /)$.

\begin{abstract}
The worldwide increase in nonalcoholic fatty liver disease (NAFLD) is a major public health problem. Obesity and diabetes are risk factors for NAFLD and the development of liver fibrosis is a risk factor for liver cancer. Periodontal disease bacteria can also exacerbate NAFLD. We previously reported that amazake, a traditional Japanese fermented food, improves the quality of life (QOL) of patients with liver cirrhosis. In this study, we investigated the effect of amazake intake on NAFLD patients with periodontal disease. Ten patients (mean age: $57.1 \pm 19.2$ years) consumed $100 \mathrm{~g}$ of amazake daily for 60 days. On days 0 and 60, their body mass index (BMI), body fat percentage, serum biochemical parameters, periodontal disease bacteria in saliva, and ten visual analog scales (VASs), namely, sense of abdomen distension, edema, fatigue, muscle cramps, loss of appetite, taste disorder, constipation, diarrhea, depression, and sleep disorder, were measured. For periodontal bacteria, the numbers of six types of bacteria in saliva (Porphyromonas gingivalis, Tannerella forsythia, Treponema denticola, Aggregatibacter actinomycetemcomitans, Prevotella intermedia, and Fusobacterium necleatum) and P. gingivalis-specific fimA genotype were determined. After 60 days of amazake consumption, eosinophils $(p<0.05)$, immune reactive insulin (IRI) $(p<0.01)$, and HOMA-IR $(p<0.05)$ had significantly increased and tumor necrosis factor $\alpha$ (TNF $\alpha)(p<0.01)$, muscle cramps $(p<0.05)$, and depression $(p<0.05)$ had significantly decreased. All subjective symptoms improved after amazake intake. No change was observed in the periodontal bacteria. In conclusion, amazake significantly decreased TNF $\alpha$ and improved the QOL of the patients with NAFLD and periodontitis. However, caution should be exercised because amazake, which is manufactured using techniques that lead to concentrations of glucose from the saccharification of rice starch, may worsen glucose metabolism in NAFLD patients. Amazake may be an effective food for improving the symptoms of a fatty liver if energy intake is regulated.
\end{abstract}

Keywords: amazake; NAFLD; periodontal disease bacteria; quality of life

\section{Introduction}

Nonalcoholic fatty liver disease (NAFLD) is a global public health issue since it is the most common liver disease worldwide [1]. In recent years, the number of patients with NAFLD has been increasing [2-4]. In Japan, the prevalence of NAFLD among screened individuals increased from $18 \%$ in 2001 [5] to $29.7 \%$ in $2009-2010$ [6]. In 2016, it was estimated that there were approximately 20 million people with NAFLD and 4 million people with non-alcoholic steatohepatitis (NASH) in Japan, and this number is expected to increase further by 2030 [7]. 
Obesity is the most important factor in the development of NAFLD/NASH [8] and weight loss through diet and exercise therapy can improve the pathogenesis $[9,10]$. The main causes of NAFLD/NASH are the exacerbation of insulin resistance, metabolic syndrome, and related diseases, such as type 2 diabetes mellitus, dyslipidemia, and hypertension, among which, type 2 diabetes mellitus is closely related to the development of NAFLD/NASH [11]. In addition, according to the results of the NASH Clinical Research Network in the United States, which assessed health-related quality of life in patients with NAFLD/NASH, patients with NAFLD had a lower quality of life (QOL) in both physical and mental aspects compared with the general population [12].

Periodontal disease is known to be associated with cardiovascular disease [13], type 2 diabetes mellitus [14], and other systemic diseases [15]. Periodontal disease homeostatically increases the production of tumor necrosis factor $\alpha(\mathrm{TNF} \alpha)$ and exacerbates the pathogenesis of diabetes mellitus through insulin resistance [16]. Among the periodontal pathogens, Porphyromonas gingivalis (P. gingivalis) was reported to infect many organs, including the brain, lungs, bronchi, liver, blood vessels, placenta, and coronary arteries [17]. P. gingivalis was shown to be a potential cause and risk factor for NAFLD/NASH [18,19]. According to Arimatsu et al., the proposed mechanisms by which periodontal disease induces systemic inflammation are as follows: (i) the direct effect of infectious agents and their products; (ii) increased expression of cytokines, chemokines, and cell adhesion molecules produced in periodontitis lesions; (iii) translocation of swallowed P. gingivalis from the gut to the circulatory system; and (iv) alteration of gut-microbial-composition-induced increases in gut epithelial permeability by swallowed $P$. gingivalis [20]. Arimatsu et al. revealed that the population of Bacteroidales in the gut microbiota was significantly elevated in mice that were administered $P$. gingivalis, which coincided with increases in insulin resistance and systemic inflammation [20]. We reported that the periodontal inflammatory response could be associated with liver fibrosis and obesity in hepatitis $C$ virus $(\mathrm{HCV})$ and hepatitis $B$ virus (HBV) patients with liver disease [21] and that the red complex (P. gingivalis, Tannerella forsythia, and Treponema denticola) that is involved in the pathogenesis of periodontal disease was associated with cirrhosis [22].

We previously reported that amazake, which is a traditional fermented beverage produced in Japan for more than several hundred years, was effective in late evening snacks (LES) therapy for patients that were infected with HCV or HBV and with cirrhosis [23]. Amazake is a nutritious drink that contains essential amino acids and vitamins, as well as a high concentration of glucose. Recently, it has also been called Japanese yogurt, and its health benefits are attracting attention [24]. Hamajima et al. revealed for the first time that glycosylceramide, which is present in large amounts in the koji used for the fermentation of amazake, has the effect of improving the profile of intestinal bacteria [25]. They showed that the koji glycosylceramide is degraded in the intestine, is absorbed and transported to the liver by lymphatic vessels, and activates peroxisome proliferator-activated receptor $\gamma(\operatorname{PPAR} \gamma)$ in hepatocytes and lowers liver cholesterol. Furthermore, Hamajima et al. showed that the koji glycosylceramide decreases liver cholesterol in obese mice by altering the expression of genes in the metabolism of cholesterol, such as cholesterol 7-alphahydroxylase (CYP7A1, which is the rate-limiting enzyme for cholesterol catabolism) and ATP binding cassette subfamily G member 8 (ABCG8, which is the protein that is implicated in the biliary excretion of cholesterol) involved in liver cholesterol metabolism [26].

There are still no drugs that are approved for NAFLD/NASH in Japan. This study was designed as a first-time, pilot study to investigate how the intake of amazake affects NAFLD patients and whether this may also indirectly affect periodontal pathogens. Hypothesizing the effect of amazake on NAFLD and periodontal pathogens, we measured the amount of periodontal bacteria in this study. The purpose of this study was to examine whether amazake consumption in NAFLD patients with periodontal disease reduces inflammatory cytokine markers and P. gingivalis levels, as well as improves the QOL. 


\section{Materials and Methods}

\subsection{Subjects}

The subjects were those who were diagnosed with a fatty liver with liver dysfunction and periodontal disease, who were visiting the Saga University Hospital, and who met the following eligibility criteria: (i) at least 20 years old at the time of obtaining consent, (ii) any gender, (iii) obtained written consent for this study, (iv) had not consumed amazake for more than one month, (v) had abnormal liver functions such as aspartate aminotransferase (AST) and alanine aminotransferase (ALT) levels in blood tests, (vi) diagnosed as NAFLD using abdominal ultrasonography, (vii) diagnosed with periodontal disease, and (viii) negative for HCV antibody and negative for hepatitis B surface antigen. NAFLD was defined as the presence of fatty deposits in the liver and the exclusion of other causes, such as alcoholic liver disease.

The exclusion criteria were (i) subjects who had not given consent, (ii) subjects who had been taking amazake continuously for more than one month, (iii) subjects who were pregnant or lactating, (iv) complications and/or history of other liver diseases (chronic hepatitis $\mathrm{C}$, chronic hepatitis $\mathrm{B}$, autoimmune hepatitis, primary biliary cirrhosis, primary sclerosing cholangitis, Wilson's disease, etc.), (v) presence of HCC (except for patients with no recurrence for more than one year after treatment for HCC), (vi) severe liver dysfunction and liver failure (encephalopathy, ascites, ruptured varicose veins, hyperbilirubinemia, etc.), (vii) diabetic patients on insulin therapy (excluding patients that managed their condition using only oral hypoglycemic agents or diet/exercise therapy with good glycemic control and $\mathrm{HbA} 1 \mathrm{c}$ below $7.0 \%$ ), (viii) severe organic passage disorders, (ix) severe renal dysfunction, (x) serum creatinine greater than or equal to $2.0 \mathrm{mg} / \mathrm{dL}$, and (xi) serum total bilirubin of $3.0 \mathrm{mg} / \mathrm{dL}$ or higher.

Prohibited concomitant medications and concomitant dietary supplements were as follows: (i) antiviral drugs for liver disease (oral direct-acting antiviral drugs "DAA," interferon, peginterferon, ribavirin, nucleic acid analog, etc.), (ii) corticosteroids, and (iii) new dietary supplements including probiotics (however, intake of dietary supplements that were taken before the study was acceptable).

A total of 11 patients with NAFLD and periodontitis were enrolled in the study at Saga University Hospital from May 2018 to November 2018, of whom, one was excluded during screening. The reason for exclusion was a contravention of the exclusion criteria. The patients ranged in age from 24 to 76 years, with an average age of $57.1 \pm 19.2$ years. The subjects' background information is shown in Table 1. The diagnosis of liver disease was based on clinical, serological, imaging, and/or histological evidence that was analyzed by a hepatologist. One case of liver cirrhosis was found among the 10 cases. The diagnosis of periodontal disease was made by an oral surgeon, who confirmed bleeding from the gingiva, plaque-stained calculus, and gingival recession. Salivary occult blood testing was performed using the commercial kit PerioScreen ${ }^{\circledR}$ (Sunstar Co. Ltd., Osaka, Japan), which uses a monoclonal antibody against human hemoglobin. Patients were followed as outpatients and no therapeutic interventions, such as lifestyle changes, physical activity, or treatment of periodontal disease, were performed after the study. One subject had a history of treatment for colorectal cancer and another had a history of treatment for breast cancer. The diagnosis of diabetes mellitus was made in accordance with the diagnostic criteria of Standards of Medical Care in Diabetes 2016 [27].

Table 1. Characteristics of the study population $(n=10)$.

\begin{tabular}{ccc}
\hline Characteristics & Variable & Total \\
\hline Sex & Male/female & $2 / 8$ \\
Age & Mean \pm SD, years & $57.1 \pm 19.2$ \\
Diagnosis of liver diseases & NAFLD & $10(100 \%)$ \\
Diabetes mellitus & Positive (\%) & $2(20 \%)$ \\
Dyslipidemia & Positive (\%) & $7(70 \%)$ \\
\hline
\end{tabular}


Table 1. Cont.

\begin{tabular}{ccc}
\hline Characteristics & Variable & Total \\
\hline Hypertension $_{\text {Depression a }}^{\text {a }}$ & Positive $(\%)$ & $4(40 \%)$ \\
History of extrahepatic malignant tumor ${ }^{b}$ & Positive $(\%)$ & $2(20 \%)$ \\
A habit of tooth brushing after meals & Positive $(\%)$ & $2(20 \%)$ \\
Anti-HCV & Positive $(\%)$ & $2(20 \%)$ \\
HBsAg & Positive (\%) & $0(0 \%)$ \\
\hline
\end{tabular}

Data are presented as $n$ or the mean \pm SD.${ }^{\text {a }}$ None of the patients were taking antidepressants. ${ }^{b}$ One patient had a history of treatment for colorectal cancer and another had a history of treatment for breast cancer. Anti-HCV, antibodies to hepatitis $\mathrm{C}$ virus; $\mathrm{HBsAg}$, hepatitis B surface antigen; SD, standard deviation.

\subsection{Study Assessment Protocol}

The intervention schedule is shown in Figure 1. Each patient drank $100 \mathrm{~g}$ of amazake (equivalent to $112 \mathrm{kcal}$ ) (Hashimoto Shoyu Co. Ltd., Kumamoto, Japan) daily for 60 days. One hundred grams of amazake contains $25.6 \mathrm{~g}$ carbohydrates, $1.7 \mathrm{~g}$ protein, $0.3 \mathrm{~g}$ fat, $0.66 \mathrm{~g}$ salt, and trace ingredients (vitamins, essential amino acids, etc.). Amazake does not contain any alcohol. The study period comprised 60 days with evaluations at baseline (visit 1) and 60 days (visit 2). During the intervention, patients were instructed to reduce their rice or snack intake by about $100 \mathrm{kcal}$ per day.

\section{Day $0 \quad$ Day 60}

\section{Amazake intake $100 \mathrm{~g}$ / day}

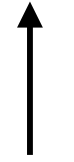

Visit 1

\section{Measurements}

- Body mass index

- Clinical and biochemical measurements

- Identification of periodontitis bacteria in saliva

- Evaluation of VAS

\section{$\uparrow$}

Visit 2

\section{Measurements}

- Body mass index

- Clinical and biochemical measurements

- Identification of periodontitis bacteria in saliva

- Evaluation of VAS

Figure 1. Study design.

On days 0 and 60, we evaluated the patients' body mass index (BMI), as well as their subjective symptoms (sense of abdomen distension, edema, fatigue, muscle cramps, loss of appetite, taste disorder, constipation, diarrhea, depression, and sleep disorder) using a visual analog scale (VAS) (Figure 2). A VAS is a horizontal line, $100 \mathrm{~mm}$ in length, anchored by word descriptors for subjective symptoms at each end. The patients were asked to mark the line at the point that they felt represented their perception of their current state. The VAS score was determined by measuring in millimeters from the left-hand end of the line to the point that the patient had marked, where $0 \mathrm{~mm}$ indicated an absence of symptoms and $100 \mathrm{~mm}$ indicated the worst symptom.

The following blood biochemical parameters were measured in all the patients on days 0 and 60: white blood cell (WBC) count, red blood cell (RBC) count, hemoglobin $(\mathrm{Hb})$, platelets (PLT) count, AST, ALT, gamma-glutamyl transpeptidase $(\gamma-\mathrm{GTP})$, lactic dehydrogenase (LDH), alkaline phosphatase (ALP), total protein, albumin, total cholesterol (T. cho), low-density lipoprotein (LDL), high-density lipoprotein (HDL), triglyceride, total 
bilirubin (T. Bil), direct bilirubin (D. Bil), creatinine (Crea), blood urea nitrogen (BUN), serum iron (Fe), ferritin, zinc, fasting blood glucose, hemoglobin A1c (HbA1c), fasting immune reactive insulin (IRI), HOMA-IR, AFP, total procollagen type 1 amino-terminal propeptide (Total P1NP), type III procollagen-N-peptide (P-III-P), type IV collagen 7s domain, mac-2 binding protein glycosylated isomers (M2BPGi) [28], tartrate-resistant acid phosphatase-5b (TRACP-5b), high molecular weight adiponectin, leptin, interleukin-6 (IL6), high-sensitivity $\mathrm{TNF} \alpha$, and high-sensitivity C-reactive protein (CRP). The formula for the HOMA-IR is as follows: HOMA-IR $=$ fasting blood glucose $\times$ fasting insulin $/ 405$.

Patient Name: $\quad$ Date:

\section{Q1. Sense of abdomen distension}

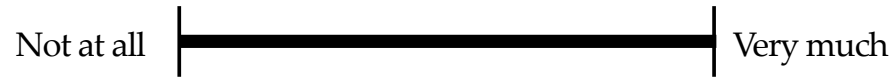

\section{Q2. Edema}

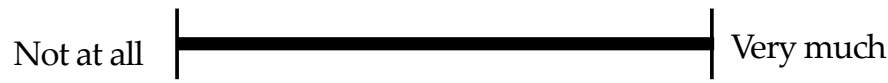

\section{Q3. Fatigue}

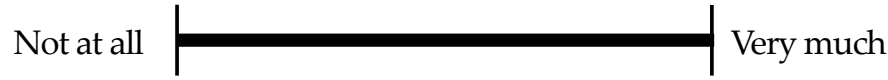

\section{Q4. Muscle cramps}

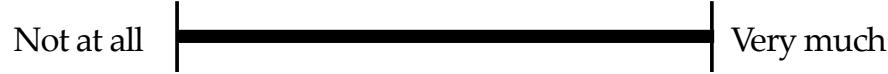

\section{Q5. Loss of appetite}

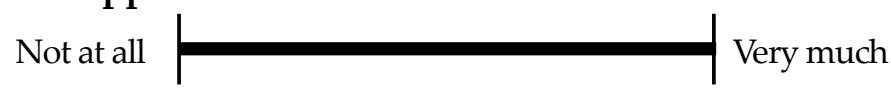

\section{Q6. Taste disorder}

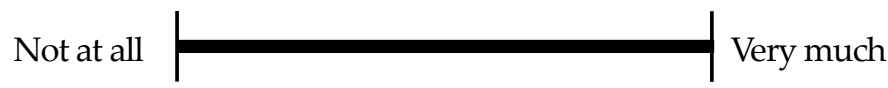

\section{Q7. Constipation}

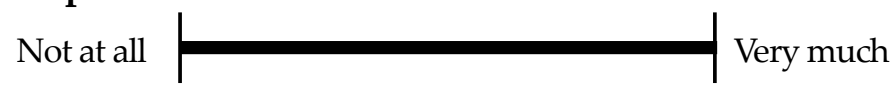

\section{Q8. Diarrhea}

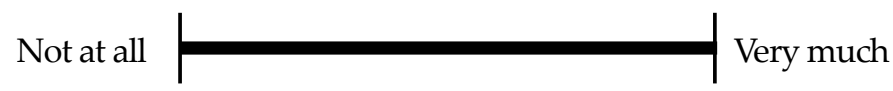

\section{Q9. Depression}

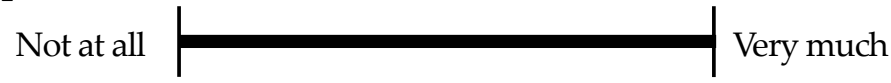

\section{Q10. Sleep disorder}

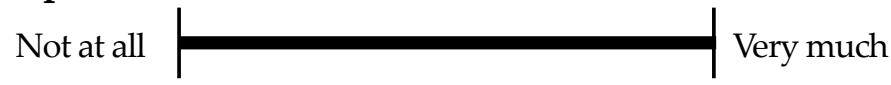

Figure 2. Visual analog scale (VAS) of 10 items. A VAS is a horizontal line, $100 \mathrm{~mm}$ in length, anchored by word descriptors at each end.

\subsection{Saliva Sample Collection}

Whole saliva $(1 \mathrm{~mL})$ was collected from the patients following chewing a non-flavored gum for $5 \mathrm{~min}$. All samples were immediately sent to the Health Examination Laboratory (BML Inc., Tokyo, Japan) for bacterial testing [29].

\subsection{Identification of Periodontitis Bacteria and Detection of P. gingivalis fimA Genotypes}

The total number of bacteria in saliva and the counts of 6 types of periodontal pathogens (Aggregatibacter actinomycetemcomitans, Prevotella intermedia, P. gingivalis, T. forsythia, T. denticola, Fusobacterium necleatum) were quantified using the modified poly- 
merase chain reaction (PCR) Invader assay, as described previously [30]. P. gingivalisspecific fim $A$ genotypes, ranging from types I-V, were detected in all patients.

\subsection{Safety Monitoring}

Safety was assessed in terms of the physical examination results, laboratory test results, and clinical adverse events. When the ten patients had completed the study, the data monitoring committee evaluated the safety and disadvantages of the subjects and confirmed that there were no severe adverse events or disadvantages observed in the subjects.

\subsection{Statistical Analysis}

All data are expressed as the number or mean \pm standard deviation. Statistical comparisons between before and 60 days after intake of amazake were performed using a paired $t$-test. A $p$-value $<0.05$ was considered to indicate a statistically significant difference. All analyses were performed using JMP 13 (SAS Institute Inc., Cary, NC, USA).

\section{Results}

\subsection{Patient Characteristics}

Patient characteristics prior to the amazake intake are summarized in Table 2. The mean values of $\mathrm{BMI}$ and body fat percentage were above normal, indicating obesity. Serum AST, ALT, $\gamma$-GTP, ferritin, HOMA-IR, P-III-P, and high-sensitivity CRP were elevated compared to normal.

Table 2. Effects of amazake intake on biochemical examinations, VASs, and periodontitis disease bacteria.

\begin{tabular}{|c|c|c|c|c|c|c|}
\hline \multirow[t]{2}{*}{ Variable } & Baseline & $\begin{array}{c}60 \text { Days after } \\
\text { Amazake Intake }\end{array}$ & \multirow[t]{2}{*}{ Difference in Means } & \multirow[t]{2}{*}{ LCL } & \multirow[t]{2}{*}{ UCL } & \multirow[t]{2}{*}{$p$-Value } \\
\hline & $($ Mean \pm SD) & $($ Mean \pm SD) & & & & \\
\hline BMI, $\mathrm{kg} / \mathrm{m}^{2}$ & $27.39 \pm 4.1$ & $27.45 \pm 4.1$ & 0.06 & -0.37 & 0.49 & NS \\
\hline Body fat percentage, $\%$ & $31.18 \pm 5.2$ & $31.99 \pm 5.6$ & 0.81 & -0.92 & 2.54 & NS \\
\hline $\mathrm{WBC}, 10^{3} / \mu \mathrm{L}$ & $5.55 \pm 1.9$ & $5.14 \pm 1.8$ & -0.41 & -1.07 & 0.25 & NS \\
\hline Neutrophil count, \% & $55.87 \pm 11.7$ & $56.85 \pm 9.5$ & 0.98 & -2.61 & 4.57 & NS \\
\hline Lymphocyte counts, \% & $33.90 \pm 10.6$ & $32.67 \pm 8.5$ & -1.23 & -4.30 & 1.84 & NS \\
\hline Monocyte count, \% & $6.85 \pm 1.7$ & $6.46 \pm 1.5$ & -0.39 & -1.44 & 0.66 & NS \\
\hline Eosinophil count, \% & $2.72 \pm 1.4$ & $3.27 \pm 1.4$ & 0.55 & 0.11 & 0.99 & 0.0203 \\
\hline Basophil count, \% & $0.66 \pm 0.3$ & $0.75 \pm 0.4$ & 0.09 & -0.06 & 0.24 & NS \\
\hline $\mathrm{RBC}, 10^{6} / \mu \mathrm{L}$ & $4.50 \pm 0.5$ & $4.49 \pm 0.5$ & -0.003 & -0.05 & 0.05 & NS \\
\hline $\mathrm{Hb}, \mathrm{g} / \mathrm{dL}$ & $14.22 \pm 1.7$ & $14.12 \pm 1.6$ & -0.10 & -0.31 & 0.10 & NS \\
\hline PLT, $10^{4} / \mu \mathrm{L}$ & $22.39 \pm 9.1$ & $21.95 \pm 9.1$ & -0.44 & -1.21 & 0.33 & NS \\
\hline AST, U/L & $47.6 \pm 28.0$ & $51.5 \pm 40.1$ & 3.9 & -7.91 & 15.71 & NS \\
\hline ALT, U/L & $58.4 \pm 38.1$ & $56.2 \pm 36.7$ & -2.2 & -13.03 & 8.63 & NS \\
\hline$\gamma-\mathrm{GTP}, \mathrm{U} / \mathrm{L}$ & $44.5 \pm 29.9$ & $47.8 \pm 33.7$ & 3.3 & -4.47 & 11.07 & NS \\
\hline LDH, U/L & $196.2 \pm 17.2$ & $200.4 \pm 26.4$ & 4.2 & -8.16 & 16.56 & NS \\
\hline ALP, U/L & $251.5 \pm 83.6$ & $252.8 \pm 82.4$ & 1.3 & -14.9 & 17.5 & NS \\
\hline Total protein, $\mathrm{g} / \mathrm{dL}$ & $7.47 \pm 0.2$ & $7.46 \pm 0.3$ & -0.01 & -0.25 & 0.23 & NS \\
\hline Albumin, g/dL & $4.26 \pm 0.3$ & $4.26 \pm 0.3$ & 0 & -0.22 & 0.22 & NS \\
\hline T. cho, mg/dL & $187.7 \pm 25.2$ & $190.3 \pm 34.6$ & 2.6 & -14.51 & 19.71 & NS \\
\hline LDL, mg/dL & $125.3 \pm 24.5$ & $126.9 \pm 31.6$ & 1.6 & -13.6 & 16.8 & NS \\
\hline $\mathrm{HDL}, \mathrm{mg} / \mathrm{dL}$ & $48.7 \pm 9.9$ & $49.6 \pm 10.1$ & 0.9 & -3.1 & 4.9 & NS \\
\hline Triglyceride, $\mathrm{mg} / \mathrm{dL}$ & $143.2 \pm 86.6$ & $130.2 \pm 51.9$ & -13 & -44.57 & 18.57 & NS \\
\hline T.Bil, mg/dL & $0.80 \pm 0.3$ & $0.85 \pm 0.3$ & 0.05 & -0.12 & 0.22 & NS \\
\hline D.Bil, mg/dL & $0.10 \pm 0$ & $0.11 \pm 0.1$ & 0.01 & -0.03 & 0.05 & NS \\
\hline Crea, mg/dL & $0.64 \pm 0.2$ & $0.63 \pm 0.2$ & -0.01 & -0.05 & 0.03 & NS \\
\hline $\mathrm{BUN}, \mathrm{mg} / \mathrm{dL}$ & $12.80 \pm 3.1$ & $13.19 \pm 2.8$ & 0.39 & -1.57 & 2.35 & NS \\
\hline $\mathrm{Fe}, \mu \mathrm{g} / \mathrm{dL}$ & $126.3 \pm 37.8$ & $117.6 \pm 25.2$ & -8.7 & -39.07 & 21.67 & NS \\
\hline Ferritin, $\mu \mathrm{g} / \mathrm{dL}$ & $231.9 \pm 206.7$ & $231.2 \pm 196.2$ & -0.7 & -22.38 & 20.98 & NS \\
\hline Zinc, $\mu \mathrm{g} / \mathrm{dL}$ & $80.6 \pm 14.6$ & $70.7 \pm 7.6$ & -9.9 & -20.74 & 0.94 & NS \\
\hline Fasting plasma glucose, $\mathrm{mg} / \mathrm{dL}$ & $108.2 \pm 19.6$ & $109.6 \pm 28.4$ & 1.4 & -14.48 & 17.28 & NS \\
\hline $\mathrm{HbA1c}, \%$ & $5.9 \pm 0.5$ & $6.1 \pm 0.7$ & 0.2 & -0.02 & 0.42 & NS \\
\hline Fasting IRI, $\mu \mathrm{U} / \mathrm{mL}$ & $16.18 \pm 7.3$ & $24.42 \pm 10.0$ & 8.24 & 2.87 & 13.61 & 0.0070 \\
\hline HOMA-IR & $4.47 \pm 2.5$ & $6.72 \pm 3.6$ & 2.25 & 0.62 & 3.87 & 0.0122 \\
\hline $\mathrm{AFP}, \mathrm{ng} / \mathrm{mL}$ & $4.54 \pm 1.6$ & $4.60 \pm 1.9$ & 0.06 & -0.88 & 1 & NS \\
\hline
\end{tabular}


Table 2. Cont.

\begin{tabular}{|c|c|c|c|c|c|c|}
\hline \multirow[t]{2}{*}{ Variable } & Baseline & $\begin{array}{c}60 \text { Days after } \\
\text { Amazake Intake }\end{array}$ & \multirow[t]{2}{*}{ Difference in Means } & \multirow[t]{2}{*}{ LCL } & \multirow[t]{2}{*}{ UCL } & \multirow[t]{2}{*}{$p$-Value } \\
\hline & $($ Mean \pm SD) & $($ Mean \pm SD) & & & & \\
\hline Total P1NP, ng/mL & $58.88 \pm 15.3$ & $54.75 \pm 19.0$ & -4.13 & -9.17 & 0.91 & NS \\
\hline P-III-P, ng/mL & $11.65 \pm 5.3$ & $11.98 \pm 5.0$ & 0.32 & -1.22 & 1.86 & NS \\
\hline $\begin{array}{l}\text { Type IV collagen 7s domain, } \\
\text { ng/mL }\end{array}$ & $5.86 \pm 2.3$ & $6.00 \pm 2.1$ & 0.14 & -0.26 & 0.54 & NS \\
\hline M2BPGi, COI & $1.61 \pm 1.3$ & $1.64 \pm 1.3$ & 0.03 & -0.23 & 0.3 & NS \\
\hline $\mathrm{TRACP}-5 \mathrm{~b}, \mathrm{mU} / \mathrm{dL}$ & $320.56 \pm 147.5$ & $354.40 \pm 140.9$ & 33.84 & -11.67 & 79.35 & NS \\
\hline $\begin{array}{l}\text { High molecular weight } \\
\text { adiponectin, } \mu \mathrm{g} / \mathrm{dL}\end{array}$ & $2.65 \pm 1.3$ & $2.68 \pm 1.5$ & 0.03 & -0.17 & 0.22 & NS \\
\hline Leptin, $\mathrm{ng} / \mathrm{mL}$ & $25.61 \pm 13.0$ & $31.09 \pm 19.9$ & 5.48 & -3.06 & 14.02 & NS \\
\hline IL-6, pg/mL & $2.16 \pm 0.8$ & $2.50 \pm 1.2$ & 0.34 & -0.35 & 1.03 & NS \\
\hline High-sensitivity TNF $\alpha, p g / m L$ & $1.41 \pm 0.4$ & $1.21 \pm 0.4$ & -0.20 & -0.31 & -0.09 & 0.0025 \\
\hline High-sensitivity CRP, ng/mL & $2344.5 \pm 2266.7$ & $2311.6 \pm 3442.9$ & -32.9 & -1191.25 & 1125.45 & NS \\
\hline $\begin{array}{c}\text { Total number of bacteria in } \\
\text { saliva, copy } / 10 \mu \mathrm{L} \\
\text { Number of } A \text {. }\end{array}$ & $\begin{array}{c}13,290,000 \pm \\
7,130,131.7\end{array}$ & $\begin{array}{c}11,560,000 \pm \\
6,794,147.8\end{array}$ & $-1,730,000$ & $-4,700,000$ & $1,246,368$ & NS \\
\hline $\begin{array}{c}\text { actinomycetemcomitans in saliva, } \\
\text { copy } / 10 \mu \mathrm{L}\end{array}$ & $<10$ & $<10$ & 0 & 0 & 0 & NS \\
\hline $\begin{array}{c}\text { Number of } P \text {. intermedia in saliva, } \\
\text { copy } / 10 \mu \mathrm{L}\end{array}$ & $17.8 \pm 22.6$ & $34.7 \pm 75.7$ & 16.9 & -21.08 & 54.88 & NS \\
\hline $\begin{array}{l}\text { Number of } P \text {. gingivalis in saliva, } \\
\text { copy } / 10 \mu \mathrm{L}\end{array}$ & $144.0 \pm 294.4$ & $54.0 \pm 113.8$ & -90 & -267.1 & 87.10 & NS \\
\hline $\begin{array}{l}\text { Number of T. forsythia in saliva, } \\
\text { copy } / 10 \mu \mathrm{L}\end{array}$ & $330.0 \pm 292.0$ & $703.5 \pm 1242$ & 373.5 & -433.98 & 1180.98 & NS \\
\hline $\begin{array}{c}\text { Number of } T \text {. denticola in saliva, } \\
\text { copy } / 10 \mu \mathrm{L}\end{array}$ & $369.0 \pm 492.1$ & $391.5 \pm 609.3$ & 22.5 & -188.40 & 233.40 & NS \\
\hline $\begin{array}{l}\text { Number of F. necleatum in saliva, } \\
\text { copy } / 10 \mu \mathrm{L}\end{array}$ & $\begin{array}{r}283,090 \pm \\
274,895.9\end{array}$ & $243,044 \pm 342,379.7$ & $-40,046$ & $-233,951$ & 153,859 & NS \\
\hline $\begin{array}{l}\text { P. gingivalis fimA genotype II, } \\
\text { positive }(\%)\end{array}$ & $2(20 \%)$ & $2(20 \%)$ & $n / \mathrm{a}$ & $n / \mathrm{a}$ & $n / \mathrm{a}$ & NS \\
\hline $\begin{array}{l}\text { P. gingivalis fimA below detection } \\
\text { sensitivity limits, positive (\%) }\end{array}$ & $8(80 \%)$ & $8(80 \%)$ & $n / \mathrm{a}$ & $n / \mathrm{a}$ & $n / \mathrm{a}$ & NS \\
\hline $\begin{array}{l}\text { Sense of abdominal distension } \\
\text { (VAS), } \mathrm{mm}\end{array}$ & $25.8 \pm 25.0$ & $18.6 \pm 28.9$ & -7.2 & -20.1 & 5.7 & NS \\
\hline Edema (VAS), mm & $27.2 \pm 30.9$ & $31.6 \pm 37.7$ & 4.4 & -6.48 & 15.28 & NS \\
\hline Fatigue (VAS), mm & $37.2 \pm 28.7$ & $36.8 \pm 29.6$ & -0.4 & -7.02 & 6.22 & NS \\
\hline Muscle cramps (VAS), mm & $24.1 \pm 29.6$ & $3.4 \pm 7.2$ & -20.7 & -39.62 & -1.78 & 0.0353 \\
\hline Loss of appetite (VAS), mm & $24.4 \pm 25.2$ & $12.1 \pm 9.8$ & -12.3 & -31.47 & 6.87 & NS \\
\hline Taste disorder (VAS), mm & $3.0 \pm 6.9$ & 0 & -3 & -7.93 & 1.93 & NS \\
\hline Constipation (VAS), mm & $31.4 \pm 30.0$ & $18.2 \pm 29.6$ & -13.2 & -29.34 & 2.94 & NS \\
\hline Diarrhea (VAS), mm & $16.6 \pm 29.8$ & $7.3 \pm 12.5$ & -9.3 & -28.42 & 9.82 & NS \\
\hline Depression (VAS), mm & $30.8 \pm 24.7$ & $18.2 \pm 21.6$ & -12.6 & -23.52 & -1.68 & 0.0282 \\
\hline Sleep disorder (VAS), $\mathrm{mm}$ & $25.3 \pm 24.2$ & $19.6 \pm 24.1$ & -5.7 & -25.06 & 13.66 & NS \\
\hline
\end{tabular}

Data are presented as $n(\%)$ or the mean \pm SD. Statistical comparisons between before and after 60 days of amazake intake were performed using $t$-tests. BMI, body mass index; WBC, white blood cell; RBC, red blood cell; Hb, hemoglobin; PLT, platelets; AST, aspartate aminotransferase; ALT, alanine aminotransferase; $\gamma$-GTP, gamma-glutamyl transpeptidase; LDH, lactic dehydrogenase; ALP, alkaline phosphatase; T. cho, total cholesterol; TG, triglyceride; T.Bil, total bilirubin; D.Bil, direct bilirubin; Crea, creatinine; BUN, blood urea nitrogen; Fe, serum iron; $\mathrm{HbA1c}$, hemoglobin A1c; IRI, immune reactive insulin; AFP, alpha-fetoprotein; Total P1NP, total procollagen type 1 amino-terminal propeptid; P-III-P, type III procollagen-N-peptide; M2BPGi, mac-2 binding protein glycosylated isomers; TRACP$5 \mathrm{~b}$, tartrate-resistant acid phosphatase-5b; IL6, interleukin-6; TNF $\alpha$, high-sensitivity tumor necrosis factor $\alpha$; CRP, C-reactive protein; VAS, visual analog scale; A. actinomycetemcomitans, Aggregatibacter actinomycetemcomitans; P. intermedia, Prevotella intermedia; P. gingivalis, Porphyromonas gingivalis; T. forsythia, Tannerella forsythia; T. denticola, Treponema denticola; F. necleatum, Fusobacterium necleatum; SD, standard deviation; LCL, Lower confidence limit; UCL, Upper confidence limit; $n / a$, not applicable; NS, not significant.

\subsection{Effects of Amazake Intake on Biochemical Examinations, VAS, and Periodontitis Disease Bacteria}

We analyzed any differences in laboratory data, VAS, and periodontitis disease bacteria before and after the intake of amazake (Table 2). After 60 days of amazake intake, eosinophils, IRI, and HOMA-IR were significantly elevated, while high-sensitivity TNF $\alpha$, muscle cramps, and depression were significantly decreased. All subjective symptoms were improved after the amazake intake. Taste disorder disappeared after 60 days of amazake intake. There were no significant changes in liver function values, such as AST and ALT; renal function values; fasting plasma glucose; $\mathrm{HbA1c}$; cholesterol; number of periodontal 
disease bacteria (A. actinomycetemcomitans, P. intermedia, P. gingivalis, T. forsythia, T. denticola, and $F$. necleatum); and $P$. gingivalis-specific fim $A$ genotypes after the amazake intake.

\subsection{M2BPGi Levels and Effects of Amazake Intake}

M2BPGi is a useful biomarker in diagnosing the fibrosis stage of the liver and assessing the risk of hepatocarcinogenesis [28]. We compared the effects of amazake by dividing the M2BPGi values into three groups: less than 1.00 cut-off index (C.O.I) (negative, group A), 1.00-3.00 C.O.I (positive 1+, group B), and more than 3.00 C.O.I (positive 2, group C) (Table 3). As shown in Table 3, eosinophils and HOMA-IR increased, and high-sensitivity $\mathrm{TNF} \alpha$, cramps, and depression decreased in all groups.

Table 3. Comparison of the effects of amazake according to M2BPGi levels: differences in biochemical tests, VAS, and periodontal disease.

\begin{tabular}{|c|c|c|c|}
\hline \multirow{3}{*}{ M2BPGi } & Group A & Group B & Group C \\
\hline & Negative $(n=5)$ & Positive $1(n=3)$ & Positive $2(n=2)$ \\
\hline & Difference in Means & Difference in Means & Difference in Means \\
\hline BMI, $\mathrm{kg} / \mathrm{m}^{2}$ & -0.08 & 0.23 & 0.15 \\
\hline Body fat percentage, $\%$ & 1.76 & 0.03 & -0.40 \\
\hline WBC, $10^{3} / \mu \mathrm{L}$ & -0.76 & 0.20 & -0.45 \\
\hline Neutrophil count, \% & -1.9 & 5.2 & 1.8 \\
\hline Lymphocyte counts, \% & 1.6 & -4.6 & -3.2 \\
\hline Monocyte count, \% & -0.2 & -1.1 & 0.2 \\
\hline Eosinophil count, \% & 0.38 & 0.57 & 0.95 \\
\hline Basophil count, \% & 0.1 & -0.1 & 0.3 \\
\hline $\mathrm{RBC}, 10^{6} / \mu \mathrm{L}$ & 0.01 & 0 & 0 \\
\hline $\mathrm{Hb}, \mathrm{g} / \mathrm{dL}$ & 0.03 & -0.2 & -0.4 \\
\hline $\mathrm{PLT}, 10^{4} / \mu \mathrm{L}$ & 0.02 & -1.7 & 0.3 \\
\hline AST, U/L & -3.8 & 15.3 & 6.0 \\
\hline ALT, U/L & -6.2 & 2.0 & 1.5 \\
\hline$\gamma-\mathrm{GTP}, \mathrm{U} / \mathrm{L}$ & 3.0 & 6.7 & -1.0 \\
\hline $\mathrm{LDH}, \mathrm{U} / \mathrm{L}$ & -1.0 & 13.7 & 3.0 \\
\hline $\mathrm{ALP}, \mathrm{U} / \mathrm{L}$ & -9.0 & 16.7 & 4.0 \\
\hline Total protein, $\mathrm{g} / \mathrm{dL}$ & 0.16 & -0.13 & -0.25 \\
\hline Albumin, g/dL & 0.08 & -0.03 & -0.15 \\
\hline T. cho, mg/dL & 5.8 & 10.0 & -16.5 \\
\hline $\mathrm{LDL}, \mathrm{mg} / \mathrm{dL}$ & 3.8 & 6.7 & -11.5 \\
\hline $\mathrm{HDL}, \mathrm{mg} / \mathrm{dL}$ & 1.8 & 1.3 & -2.0 \\
\hline Triglyceride, mg/dL & -23.0 & -9.7 & 7.0 \\
\hline T.Bil, mg/dL & 0.08 & -0.03 & 0.10 \\
\hline D.Bil, $\mathrm{mg} / \mathrm{dL}$ & 0 & 0.03 & 0 \\
\hline Crea, mg/dL & 0 & -0.02 & -0.03 \\
\hline $\mathrm{BUN}, \mathrm{mg} / \mathrm{dL}$ & 0.92 & 1.93 & -3.25 \\
\hline $\mathrm{Fe}, \mu \mathrm{g} / \mathrm{dL}$ & -21.2 & 4.0 & 3.5 \\
\hline Ferritin, $\mu \mathrm{g} / \mathrm{dL}$ & -15.2 & 20.3 & 4.0 \\
\hline Zinc, $\mu \mathrm{g} / \mathrm{dL}$ & -6.6 & -18.3 & -5.5 \\
\hline Fasting plasma glucose, $\mathrm{mg} / \mathrm{dL}$ & -3.4 & 1.7 & 13.0 \\
\hline $\mathrm{HbA} 1 \mathrm{c}, \%$ & 0.1 & 0.1 & 0.7 \\
\hline Fasting IRI, $\mu \mathrm{U} / \mathrm{mL}$ & 8.98 & 12.57 & -0.10 \\
\hline HOMA-IR & 2.06 & 3.29 & 1.15 \\
\hline $\mathrm{AFP}, \mathrm{ng} / \mathrm{mL}$ & -0.10 & 0.57 & -0.30 \\
\hline Total P1NP, ng/mL & -8.48 & 0.47 & -0.15 \\
\hline P-III-P, ng/mL & -0.17 & 2.56 & -1.80 \\
\hline Type IV collagen $7 \mathrm{~s}$ domain, $\mathrm{ng} / \mathrm{mL}$ & 0.26 & 0 & 0.05 \\
\hline TRACP-5b, $\mathrm{mU} / \mathrm{dL}$ & 68.88 & 4.00 & -9.00 \\
\hline
\end{tabular}


Table 3. Cont.

\begin{tabular}{|c|c|c|c|}
\hline \multirow{3}{*}{ M2BPGi } & Group A & Group B & Group C \\
\hline & Negative $(n=5)$ & Positive $1(n=3)$ & Positive $2(n=2)$ \\
\hline & Difference in Means & Difference in Means & Difference in Means \\
\hline High molecular weight adiponectin, $\mu \mathrm{g} / \mathrm{dL}$ & -0.06 & 0.31 & -0.18 \\
\hline Leptin, $\mathrm{ng} / \mathrm{mL}$ & 5.40 & 11.27 & -3.00 \\
\hline IL-6, pg/mL & -0.30 & 0.77 & 1.30 \\
\hline High-sensitivity TNF $\alpha, \mathrm{pg} / \mathrm{mL}$ & -0.24 & -0.17 & -0.16 \\
\hline High-sensitivity CRP, ng/mL & 305.40 & -208.00 & -616.00 \\
\hline Total number of bacteria in saliva, copy $/ 10 \mu \mathrm{L}$ & $-1,220,000$ & $-2,533,333$ & $-1,800,000$ \\
\hline $\begin{array}{c}\text { Number of A. actinomycetemcomitans in saliva, } \\
\text { copy } / 10 \mu \mathrm{L}\end{array}$ & 0 & 0 & 0 \\
\hline Number of $P$. intermedia in saliva, copy $/ 10 \mu \mathrm{L}$ & 0.2 & 0 & 84 \\
\hline Number of $P$. gingivalis in saliva, copy $/ 10 \mu \mathrm{L}$ & -18 & -263 & -10 \\
\hline Number of T. forsythia, in saliva, copy $/ 10 \mu \mathrm{L}$ & 760 & -20 & -3 \\
\hline Number of T. denticola in saliva, copy $/ 10 \mu \mathrm{L}$ & -14 & 96 & 4 \\
\hline Number of F. necleatum in saliva, copy $/ 10 \mu \mathrm{L}$ & 64,200 & $-216,567$ & $-35,880$ \\
\hline P. gingivalis fimA genotype II, positive (\%) & $n / \mathrm{a}$ & $n / \mathrm{a}$ & $n / \mathrm{a}$ \\
\hline $\begin{array}{l}\text { P. gingivalis fimA below detection sensitivity } \\
\text { limits, positive (\%) }\end{array}$ & $n / \mathrm{a}$ & $n / \mathrm{a}$ & $n / \mathrm{a}$ \\
\hline Sense of abdominal distension (VAS), $\mathrm{mm}$ & -15.2 & -8.3 & 14.5 \\
\hline Edema (VAS), $\mathrm{mm}$ & 9.4 & 7.7 & -13.0 \\
\hline Fatigue (VAS), mm & 2.6 & -1.7 & -6.0 \\
\hline Muscle cramps (VAS), mm & -11.4 & -22.3 & -41.5 \\
\hline Loss of appetite (VAS), $\mathrm{mm}$ & -13.6 & 3.0 & -32.0 \\
\hline Taste disorder (VAS), mm & -5.4 & -1.0 & 0 \\
\hline Constipation (VAS), mm & -28.8 & 1.3 & 4.0 \\
\hline Diarrhea (VAS), mm & -17.8 & -1.3 & 0 \\
\hline Depression (VAS), mm & -16.0 & -13.0 & -3.5 \\
\hline Sleep disorder (VAS), $\mathrm{mm}$ & 1.2 & -14.3 & -10.0 \\
\hline
\end{tabular}

BMI, body mass index; WBC, white blood cell; RBC, red blood cell; Hb, hemoglobin; PLT, platelets; AST, aspartate aminotransferase; ALT, alanine aminotransferase; $\gamma$-GTP, gamma-glutamyl transpeptidase; LDH, lactic dehydrogenase; ALP, alkaline phosphatase; T. cho, total cholesterol; TG, triglyceride; T.Bil, total bilirubin; D.Bil, direct bilirubin; Crea, creatinine; BUN, blood urea nitrogen; Fe, serum iron; HbA1c, hemoglobin A1c; IRI, immune reactive insulin; AFP, alpha-fetoprotein; Total P1NP, total procollagen type 1 amino-terminal propeptid; P-III-P, type III procollagen- $n$-peptide; M2BPGi, mac-2 binding protein glycosylated isomers; TRACP-5b, tartrate-resistant acid phosphatase-5b; IL6, interleukin-6; TNF $\alpha$, high-sensitivity tumor necrosis factor $\alpha$; CRP, C-reactive protein; VAS, visual analog scale; A. actinomycetemcomitans, Aggregatibacter actinomycetemcomitans; P. intermedia, Prevotella intermedia; P. gingivalis, Porphyromonas gingivalis; T. forsythia, Tannerella forsythia; T. denticola, Treponema denticola; F. necleatum, Fusobacterium necleatum; $n$ /a, not applicable.

\subsection{Adverse Events}

No adverse events other than elevated IRI or HOMA-IR were observed. All patients completed the intake of amazake as scheduled.

\section{Discussion}

This study investigated the effect of amazake consumption on NAFLD patients with periodontal disease. After 10 patients consumed $100 \mathrm{~g}$ of amazake daily for 60 days, eosinophils $(p<0.05)$, IRI $(p<0.01)$, and HOMA-IR $(p<0.05)$ were significantly elevated, and TNF $\alpha(p<0.01)$, muscle cramps $(p<0.05)$, and depression $(p<0.05)$ were significantly decreased. This result showed a similar tendency in cases with advanced liver fibrosis, as seen in the subgroups in Table 3. However, since the number of cases was small, the results of statistical tests should be interpreted with caution.

Amazake is a traditional Japanese sweetened beverage that is made from rice, malted rice, and water. The culture of drinking amazake dates back to the early 7 th century and is mentioned in the "Nihon Shoki," the second-oldest book of classical Japanese history. The drink was commonly consumed during the Edo period (1603-1868 AD) [31,32]. Koji amazake, a sweet non-alcoholic beverage, is made by the fermentation of rice by filamentous fungi called Aspergillus oryzae, which is known as "koji" in Japanese. In recent years, various effects of rice malt and sake lees, which are ingredients of amazake, 
were reported as follows: improvement of the intestinal environment [25,33], obesity control [34,35], suppression of cholesterol increase [36], suppression of blood pressure increase [37], prevention of amnesia [34], a whitening effect [38], a moisturizing effect [39], an effect on the elasticity of the skin [40], increased bifidobacteria in the intestine with acid protease derived from koji mold [41], and improved bowel movement in healthy people [42].

In the present study, amazake intake increased eosinophils, IRI, and HOMA-IR, while high-sensitivity $\mathrm{TNF} \alpha$, muscle cramps, and depression were significantly decreased. Amazake intake did not cause increases in fasting blood glucose and HbA1c. However, increased IRI and HOMA-IR indicate that amazake intake may have worsened insulin resistance and glucose metabolism. Because the concentration of glucose contained in amazake can be regulated by manufacturing procedures, such as via the saccharifying time, temperature, and the extent and form of propagation of the fungus Aspergillus oryzae, the glucose concentration of amazake should be decreased by manufacturers to attain its health benefits.

The fact that TNF $\alpha$ was significantly reduced by amazake intake suggested an improvement in the pathogenesis of fatty liver with periodontitis, although the mechanism is unknown. Inflammatory cytokines, such as TNF $\alpha$, IL-6, and CRP, are significantly elevated in obesity and visceral adiposity [43] and are associated with insulin resistance [44]. TNF $\alpha$ was reported to be associated with the development and progression of NAFLD [45,46] and Crespo et al. reported that the expression of TNF receptor 1 was increased in liver tissue in NASH, especially in patients with advanced fibrosis [47].

Periodontitis is the most common infectious disease worldwide [48]. Periodontal pockets are inhabited by periodontopathogenic bacteria, such as A. actinomycetemcomitans, P. intermedia, P. gingivalis, T. forsythia, T. denticola, and F. necleatum [49]. Yoneda et al. reported that a high rate of $P$. gingivalis was detected in the oral cavities of NASH patients and that the removal of $P$. gingivalis via dental treatment improved AST and ALT levels [18]. It was reported that $P$. gingivalis infection exacerbates fibrosis in NASH by stimulating the activation of hepatic stellate cells (HSCs) through the production of transforming growth factor $\beta 1$ (TGF- $\beta 1$ ) and galectin-3 [50]. P. gingivalis fimA fimbriae are classified into six genotypes (types I-V and Ib) [51], with types II/IV/Ib being invasive and types I/III/V being non-invasive [52]. In this study, there was no change in the number of periodontal bacteria and fim $A$ genotype due to amazake, but the two patients with fim $A$ both had type II, which is associated with liver fibrosis [21,22].

The fact that amazake consumption significantly elevated eosinophils may be an effective phenomenon for a fatty liver with obesity. Recently, eosinophils were reported to play a protective role against obesity [53,54]. In 2011, Wu et al. first reported on the potential of eosinophils to prevent obesity [53], feeding a high-fat diet to transgenic C57BL $/ 6$ mice lacking eosinophils ( $\triangle$ dblGATA mice) resulted in increased body fat and worsened insulin resistance in adipose tissue compared with wild-type mice. On the other hand, they showed that mice with increased eosinophils and mice with eosinophils that were increased physiologically by infection with helminths had reduced body fat gain and improved insulin sensitivity and glucose tolerance. These results suggest that eosinophils play an important role in the regulation of glucose metabolism by maintaining the function of endogenous macrophages in adipose tissue.

This study showed that the intake of amazake improved subjective symptoms, especially muscle cramps and depression, in all patients. Muscle cramps are a common comorbidity of chronic liver disease and were reported to be associated with female sex, diabetes and chronic kidney disease in chronic liver disease, and with decreased muscle mass in NAFLD [55]. Mehta et al. reported that muscle cramps are common in patients with liver disease and adversely influence their QOL [56]. Increased TNF $\alpha$ is known to be associated with muscle damage and inflammation processes in the extremities [57], as well as depression $[58,59]$. Although the precise mechanism by which amazake improved QOL is not clear, in the present study, the reduction in TNF $\alpha$ by amazake seems to have 
improved muscle cramps and depressed moods. Another reason may be that the BCAAs contained in amazake may have influenced the results [60-65]. The administration of BCAA supplements might reduce the frequency of muscle cramps in patients with cirrhosis $[66,67]$. Indeed, our previous study on the efficacy of amazake as an LES therapy in cirrhotic patients infected with HCV and HBV ameliorated muscle cramps [23].

The transfer of intestinal bacteria and their metabolites to the liver via the portal vein contributes to the pathogenesis of NAFLD. In patients with NAFLD, changes in the intestinal microbiota were shown to be closely related to the pathogenesis of the disease, especially an increase in Firmicutes [68] and a decrease in Bacteroidetes [69]. It was also reported that patients with NAFLD not only have changes in the composition of the intestinal microbiota but also increased intestinal permeability [70]. It was confirmed that ingestion of the glycosylceramide contained in Aspergillus oryzae improved the intestinal microbiota of mice, including an increase in Bacteroidetes [25]. Therefore, as a mechanism, the intake of amazake containing glycosylceramide to NAFLD patients may have induced changes in the intestinal microbiota, although the details are unknown.

The present study has several limitations. First, the sample size was small. Second, the study was not conducted as a controlled trial. Large-scale case-control studies are needed in the future to clarify the effects of amazake. Third, no assessment of periodontitis, such as measuring the probing depth or bleeding on probing, was conducted.

\section{Conclusions}

In this pilot study, it was confirmed that consuming $100 \mathrm{~g} /$ day of amazake made from rice malt for 60 days improved TNF $\alpha$ and subjective symptoms, although it exacerbated IRI and HOMA-IR in patients with NAFLD and periodontal disease. It should be emphasized that due to the small sample size in this study, these observations require further study. Because NAFLD patients often have complications of diabetes and obesity, it is desirable to consume amazake with an adequate adjustment to their energy intake.

Author Contributions: Y.N. designed the study, interpreted the data, and wrote the manuscript. H.T. collected data from participants. A.K. performed the statistical analysis. H.K. conducted the monitoring to assess the study's safety. All authors have read and agreed to the published version of the manuscript.

Funding: The present study was supported in part by the Japan Society for the Promotion of Science (JSPS) KAKENHI Grant-in-Aid for Scientific Research (C) grant numbers JP17K12012 and JP21K10263.

Institutional Review Board Statement: The study was conducted according to the guidelines of the Declaration of Helsinki, and approved by the Ethical Committee of Saga University (protocol code 2017-04-01; UMIN 000030597), on 3 July 2017.

Informed Consent Statement: Written informed consent was obtained from all subjects involved in the study.

Data Availability Statement: All the datasets generated and analyzed in the present study are included in this published article.

Acknowledgments: We would like to thank Yuichiro Eguchi (Loco Medical General Institute) for their advice and Maki Miyahara (Saga University Hospital) for their support during data collection. We would also like to thank Yasutaka Hashimoto, Kazuhiko Hashimoto, and Mayumi Hashimoto Tanaka (Hashimoto Shoyu Co. Ltd., Kumamoto, Japan) for their helpful discussions and support for this research.

Conflicts of Interest: The authors declare no conflict of interest.

\section{Abbreviations}

The abbreviations including in the text are reported alphabetically. 


\begin{tabular}{|c|c|}
\hline AFP & $\alpha$-fetoprotein \\
\hline ALP & alkaline phosphatase \\
\hline ALT & alanine aminotransferase \\
\hline Anti-HCV & antibodies to $\mathrm{HCV}$ \\
\hline AST & aspartate aminotransferase \\
\hline BCAA & branched-chain amino acids \\
\hline BMI & body mass index \\
\hline BUN & blood urea nitrogen \\
\hline Crea & creatinine \\
\hline CRP & C-reactive protein \\
\hline D.Bil & direct bilirubin \\
\hline DAA & direct-acting antiviral drug \\
\hline $\mathrm{Hb}$ & hemoglobin \\
\hline $\mathrm{HbA1c}$ & hemoglobin A1c \\
\hline HBsAg & hepatitis B surface antigen \\
\hline $\mathrm{HBV}$ & hepatitis $B$ virus \\
\hline $\mathrm{HCC}$ & hepatocellular carcinoma \\
\hline $\mathrm{HCV}$ & hepatitis $C$ virus \\
\hline HDL & high-density lipoprotein \\
\hline IL6 & interleukin-6 \\
\hline IRI & immune reactive insulin \\
\hline LDH & lactic dehydrogenase \\
\hline LDL & low-density lipoprotein \\
\hline LES & late evening snacks \\
\hline M2BPGi & mac-2 binding protein glycosylated isomers \\
\hline NAFLD & nonalcoholic fatty liver disease \\
\hline NASH & nonalcoholic steatohepatitis \\
\hline P-III-P & type III procollagen-N-peptide \\
\hline PLT & platelets \\
\hline QOL & quality of life \\
\hline RBC & red blood cell \\
\hline T. Bil & total bilirubin \\
\hline T. cho & total cholesterol \\
\hline $\mathrm{TNF} \alpha$ & tumor necrosis factor $\alpha$ \\
\hline Total P1NP & total procollagen type 1 amino-terminal propeptide \\
\hline TRACP-5b & tartrate-resistant acid phosphatase- $5 b$ \\
\hline VAS & visual analog scale \\
\hline WBC & white blood cell \\
\hline$\gamma$-GTP & gamma-glutamyl transpeptidase \\
\hline
\end{tabular}

\section{References}

1. Younossi, Z.M.; Otgonsuren, M.; Henry, L.; Venkatesan, C.; Mishra, A.; Erario, M.; Hunt, S. Association of nonalcoholic fatty liver disease (NAFLD) with hepatocellular carcinoma (HCC) in the United States from 2004 to 2009. Hepatology 2015, 62, 1723-1730. [CrossRef]

2. Younossi, Z.M.; Koenig, A.B.; Abdelatif, D.; Fazel, Y.; Henry, L.; Wymer, M. Global epidemiology of nonalcoholic fatty liver disease-Meta-analytic assessment of prevalence, incidence, and outcomes. Hepatology 2015, 64, 73-84. [CrossRef]

3. Estes, C.; Razavi, H.; Loomba, R.; Younossi, Z.; Sanyal, A.J. Modeling the epidemic of nonalcoholic fatty liver disease demonstrates an exponential increase in burden of disease. Hepatology 2018, 67, 123-133. [CrossRef]

4. Younossi, Z.M.; Golabi, P.; de Avila, L.; Paik, J.M.; Srishord, M.; Fukui, N.; Qiu, Y.; Burns, L.; Afendy, A.; Nader, F. The global epidemiology of NAFLD and NASH in patients with type 2 diabetes: A systematic review and meta-analysis. J. Hepatol. 2019, 71, 793-801. [CrossRef] [PubMed]

5. Hamaguchi, M.; Kojima, T.; Takeda, N.; Nakagawa, T.; Taniguchi, H.; Fujii, K.; Omatsu, T.; Nakajima, T.; Sarui, H.; Shimazaki, M.; et al. The Metabolic Syndrome as a Predictor of Nonalcoholic Fatty Liver Disease. Ann. Intern. Med. 2005, 143, 722-728. [CrossRef]

6. Eguchi, Y.; Nafld, J.-; Hyogo, H.; Ono, M.; Mizuta, T.; Ono, N.; Fujimoto, K.; Chayama, K.; Saibara, T. Prevalence and associated metabolic factors of nonalcoholic fatty liver disease in the general population from 2009 to 2010 in Japan: A multicenter large retrospective study. J. Gastroenterol. 2012, 47, 586-595. [CrossRef] [PubMed] 
7. $\quad$ Estes, C.; Anstee, Q.M.; Arias-Loste, M.T.; Bantel, H.; Bellentani, S.; Caballeria, J.; Colombo, M.; Craxi, A.; Crespo, J.; Day, C.P.; et al. Modeling NAFLD disease burden in China, France, Germany, Italy, Japan, Spain, United Kingdom, and United States for the period 2016-2030. J. Hepatol. 2018, 69, 896-904. [CrossRef] [PubMed]

8. Koda, M.; Kawakami, M.; Murawaki, Y.; Senda, M. The impact of visceral fat in nonalcoholic fatty liver disease: Cross-sectional and longitudinal studies. J. Gastroenterol. 2007, 42, 897-903. [CrossRef]

9. Thoma, C.; Day, C.P.; Trenell, M. Lifestyle interventions for the treatment of non-alcoholic fatty liver disease in adults: A systematic review. J. Hepatol. 2012, 56, 255-266. [CrossRef]

10. Glass, L.M.; Dickson, R.C.; Anderson, J.C.; Suriawinata, A.A.; Putra, J.; Berk, B.S.; Toor, A. Total Body Weight Loss of $\geq 10 \%$ Is Associated with Improved Hepatic Fibrosis in Patients with Nonalcoholic Steatohepatitis. Dig. Dis. Sci. 2015, 60, 1024-1030. [CrossRef]

11. El-Serag, H.B.; Tran, T.; Everhart, J.E. Diabetes increases the risk of chronic liver disease and hepatocellular carcinoma. Gastroenterology 2004, 126, 460-468. [CrossRef] [PubMed]

12. David, K.; Kowdley, K.V.; Unalp, A.; Kanwal, F.; Brunt, E.M.; Schwimmer, J.B.; NASH CRN Research Group. Quality of life in adults with nonalcoholic fatty liver disease: Baseline data from the nonalcoholic steatohepatitis clinical research network. Hepatology 2009, 49, 1904-1912. [CrossRef]

13. Beck, J.D.; Slade, G.; Offenbacher, S. Oral disease, cardiovascular disease and systemic inflammation. Periodontol. 2000 2000, 23, 110-120. [CrossRef] [PubMed]

14. Mealey, B.L.; Oates, T.W.; American Academy of Periodontology. Diabetes Mellitus and Periodontal Diseases. J. Periodontol. 2006, 77, 1289-1303. [CrossRef] [PubMed]

15. Falcao, A.; Bullón, P. A review of the influence of periodontal treatment in systemic diseases. Periodontol. 2000 2019, 79, 117-128. [CrossRef] [PubMed]

16. Mealey, B.L.; Rethman, M.P. Periodontal disease and diabetes mellitus. Bidirectional relationship. Dent. Today 2003, 22, 107-113. [PubMed]

17. Mei, F.; Xie, M.; Huang, X.; Long, Y.; Lu, X.; Wang, X.; Chen, L. Porphyromonas gingivalis and Its Systemic Impact: Current Status. Pathogens 2020, 9, 944. [CrossRef]

18. Yoneda, M.; Naka, S.; Nakano, K.; Wada, K.; Endo, H.; Mawatari, H.; Imajo, K.; Nomura, R.; Hokamura, K.; Ono, M.; et al. Involvement of a periodontal pathogen, Porphyromonas gingivalis on the pathogenesis of non-alcoholic fatty liver disease. BMC Gastroenterol. 2012, 12, 16. [CrossRef]

19. Alazawi, W.; Bernabe, E.; Tai, D.; Janicki, T.; Kemos, P.; Samsuddin, S.; Syn, W.-K.; Gillam, D.; Turner, W. Periodontitis is associated with significant hepatic fibrosis in patients with non-alcoholic fatty liver disease. PLoS ONE 2017, 12, e0185902. [CrossRef]

20. Arimatsu, K.; Yamada, H.; Miyazawa, H.; Minagawa, T.; Nakajima, M.; Ryder, M.I.; Gotoh, K.; Motooka, D.; Nakamura, S.; Iida, T.; et al. Oral pathobiont induces systemic inflammation and metabolic changes associated with alteration of gut microbiota. Sci. Rep. 2015, 4, 4828. [CrossRef]

21. Nagao, Y.; Kawahigashi, Y.; Sata, M. Association of Periodontal Diseases and Liver Fibrosis in Patients With HCV and/or HBV infection. Zahedan J. Res. Med Sci. 2014, 14, e23264. [CrossRef]

22. Nagao, Y.; Tanigawa, T. Red complex periodontal pathogens are risk factors for liver cirrhosis. Biomed. Rep. 2019, 11, 199-206. [CrossRef] [PubMed]

23. Sata, Y.N.A.M. Effect of a Late Evening Snack of Amazake in Patients with Liver Cirrhosis: A Pilot Study. J. Nutr. Food Sci. 2013, 3, 203. [CrossRef]

24. Maruki-Uchida, H.; Sai, M.; Yano, S.; Morita, M.; Maeda, K. Amazake made from sake cake and rice koji suppresses sebum content in differentiated hamster sebocytes and improves skin properties in humans. Biosci. Biotechnol. Biochem. 2020, 84, 1689-1695. [CrossRef] [PubMed]

25. Hamajima, H.; Matsunaga, H.; Fujikawa, A.; Sato, T.; Mitsutake, S.; Yanagita, T.; Nagao, K.; Nakayama, J.; Kitagaki, H. Erratum to: Japanese traditional dietary fungus koji Aspergillus oryzae functions as a prebiotic for Blautia coccoides through glycosylceramide: Japanese dietary fungus koji is a new prebiotic. SpringerPlus 2016, 5, 1869. [CrossRef] [PubMed]

26. Hamajima, H.; Tanaka, M.; Miyagawa, M.; Sakamoto, M.; Nakamura, T.; Yanagita, T.; Nishimukai, M.; Mitsutake, S.; Nakayama, J.; Nagao, K.; et al. Koji glycosylceramide commonly contained in Japanese traditional fermented foods alters cholesterol metabolism in obese mice. Biosci. Biotechnol. Biochem. 2019, 83, 1514-1522. [CrossRef]

27. American Diabetes Association 2. Classification and Diagnosis of Diabetes. Diabetes Care 2016, 39, S13-S22. [CrossRef]

28. Kamada, Y.; Ono, M.; Hyogo, H.; Fujii, H.; Sumida, Y.; Mori, K.; Tanaka, S.; Yamada, M.; Akita, M.; Mizutani, K.; et al. A novel noninvasive diagnostic method for nonalcoholic steatohepatitis using two glycobiomarkers. Hepatology 2015, 62, 1433-1443. [CrossRef]

29. Tada, A.; Takeuchi, H.; Shimizu, H.; Tadokoro, K.; Tanaka, K.; Kawamura, K.; Yamaguchi, T.; Egashira, T.; Nomura, Y.; Hanada, N. Quantification of Periodontopathic Bacteria in Saliva Using the Invader Assay. Jpn. J. Infect. Dis. 2012, 65, 415-423. [CrossRef]

30. Tadokoro, K.; Yamaguchi, T.; Kawamura, K.; Shimizu, H.; Egashira, T.; Minabe, M.; Yoshino, T.; Oguchi, H. Rapid quantification of periodontitis-related bacteria using a novel modification of Invader PLUS technologies. Microbiol. Res. 2010, 165, 43-49. [CrossRef]

31. Matsumoto, T. Sake in the 'Engishiki'. J. Brew. Soc. Jpn. 1981, 76, 460-465. (In Japanese) [CrossRef]

32. Koizumi, T. Food and Japanese wisdom. JJRM 2013, 61, 838-839. (In Japanese) [CrossRef] 
33. Yang, Y.; Sitanggang, N.V.; Kato, N.; Inoue, J.; Murakami, T.; Watanabe, T.; Iguchi, T.; Okazaki, Y. Beneficial effects of protease preparations derived from Aspergillus on the colonic luminal environment in rats consuming a high-fat diet. Biomed. Rep. 2015, 3, 715-720. [CrossRef] [PubMed]

34. Oura, S.; Suzuki, S.; Hata, Y.; Kawato, A.; Abe, Y. Evaluation of physiological functionalities of amazake in mice. J. Brew. Soc. Jpn. 2007, 102, 781-788. (In Japanese) [CrossRef]

35. Yoshizaki, Y.; Kawasaki, C.; Cheng, K.-C.; Ushikai, M.; Amitani, H.; Asakawa, A.; Okutsu, K.; Sameshima, Y.; Takamine, K.; Inui, A. Ricekojireduced body weight gain, fat accumulation, and blood glucose level in high-fat diet-induced obese mice. Peer 2014, 2, e540. [CrossRef]

36. Ashida, Y.; Saio, Y.; Kawato, A.; Suginami, K.; Imayasu, S. Effects of dietary sake cake on cholesterol metabolism in rats. Nippon Nogeikagaku Kaishi 1997, 71, 137-143. (In Japanese) [CrossRef]

37. Kikushima, K.; Nakagawa, T.; Shimizu, K.; Ohnuki, K. Regular intake of Japanese traditional rice fermented beverage, koji amazake for 4 weeks decreases systolic blood pressure. Jpn. Pharmacol. Ther. 2020, 48, 305-312. Available online: http: //www.pieronline.jp/content/article/0386-3603/48020/305 (accessed on 29 September 2021).

38. Jeon, H.J.; Noda, M.; Maruyama, M.; Matoba, Y.; Kumagai, A.T.; Sugiyama, M. Identification and Kinetic Study of Tyrosinase Inhibitors Found in Sake Lees. J. Agric. Food Chem. 2006, 54, 9827-9833. [CrossRef]

39. Duan, J.; Sugawara, T.; Hirose, M.; Aida, K.; Sakai, S.; Fujii, A.; Hirata, T. Dietary sphingolipids improve skin barrier functions via the upregulation of ceramide synthases in the epidermis. Exp. Dermatol. 2012, 21, 448-452. [CrossRef]

40. Shimotsuma, S.; Yamamoto, T.; Mori, S.; Morita, M.; Yano, S.; Maeda, K. Effects of oral intake of rice koji amazake on skin condition -A randomized, double-blind, placebo-controlled, parallel-group, comparison study. Jpn. Pharmacol. Ther. 2019, 47, 759-765. Available online: http:/ / www.pieronline.jp/content/article/0386-3603/47080/1269 (accessed on 29 September 2021). (In Japanese).

41. Yang, Y.; Iwamoto, A.; Kumrungsee, T.; Okazaki, Y.; Kuroda, M.; Yamaguchi, S.; Kato, N. Consumption of an acid protease derived from Aspergillus oryzae causes bifidogenic effect in rats. Nutr. Res. 2017, 44, 60-66. [CrossRef] [PubMed]

42. Mori, S.; Tanaka, Y.; Watabe, K.; Yamada, M.; Morita, M.; Matsuike, T. Amazake using the lees and rice koji promotes regular bowel movements -A randomized, placebo-controlled parallel-group comparison study. Jpn. Pharmacol. Ther. 2019, 47, 759-765. Available online: http:/ / www.pieronline.jp/content/article/0386-3603/47050/759 (accessed on 29 September 2021). (In Japanese).

43. Park, H.S.; Park, J.Y.; Yu, R. Relationship of obesity and visceral adiposity with serum concentrations of CRP, TNF- $\alpha$ and IL-6. Diabetes Res. Clin. Pract. 2005, 69, 29-35. [CrossRef]

44. Hotamisligil, G.S.; Peraldi, P.; Budavari, A.; Ellis, R.; White, M.F.; Spiegelman, B.M. IRS-1-Mediated Inhibition of Insulin Receptor Tyrosine Kinase Activity in TNF-alpha- and Obesity-Induced Insulin Resistance. Science 1996, 271, 665-670. [CrossRef] [PubMed]

45. Valenti, L.; Fracanzani, A.L.; Dongiovanni, P.; Santorelli, G.; Branchi, A.; Taioli, E.; Fiorelli, G.; Fargion, S. Tumor necrosis factor $\alpha$ promoter polymorphisms and insulin resistance in nonalcoholic fatty liver disease. Gastroenterology 2002, 122, 274-280. [CrossRef] [PubMed]

46. Wang, J.-K.; Feng, Z.-W.; Li, Y.-C.; Li, Q.-Y.; Tao, X.-Y. Association of tumor necrosis factor- $\alpha$ gene promoter polymorphism at sites -308 and -238 with non-alcoholic fatty liver disease: A meta-analysis. J. Gastroenterol. Hepatol. 2012, 27, 670-676. [CrossRef] [PubMed]

47. Crespo, J.; Cayón, A.; Fernández-Gil, P.; Hernandez-Guerra, M.; Mayorga, M.; Domínguez-Díez, A.; Fernández-Escalante, J.C.; Pons-Romero, F. Gene expression of tumor necrosis factor [alpha ] and TNF-receptors, p55 and p75, in nonalcoholic steatohepatitis patients. Hepatology 2001, 34, 1158-1163. [CrossRef] [PubMed]

48. Batchelor, P. Is periodontal disease a public health problem? Br. Dent. J. 2014, 217, 405-409. [CrossRef]

49. Socransky, S.S.; Haffajee, A.D.; Cugini, M.A.; Smith, C.; Kent, R.L., Jr. Microbial complexes in subgingival plaque. J. Clin. Periodontol. 1998, 25, 134-144. [CrossRef]

50. Nagasaki, A.; Sakamoto, S.; Chea, C.; Ishida, E.; Furusho, H.; Fujii, M.; Takata, T.; Miyauchi, M. Odontogenic infection by Porphyromonas gingivalis exacerbates fibrosis in NASH via hepatic stellate cell activation. Sci. Rep. 2020, 10, 4134. [CrossRef] [PubMed]

51. Hamada, S.; Fujiwara, T.; Morishima, S.; Takahashi, I.; Nakagawa, I.; Kimura, S.; Ogawa, T. Molecular and Immunological Characterization of the Fimbriae of Porphyromonas gingivalis. Microbiol. Immunol. 1994, 38, 921-930. [CrossRef] [PubMed]

52. Amano, A.; Nakagawa, I.; Kataoka, K.; Morisaki, I.; Hamada, S. Distribution of Porphyromonas gingivalis Strains with fimA Genotypes in Periodontitis Patients. J. Clin. Microbiol. 1999, 37, 1426-1430. [CrossRef] [PubMed]

53. Wu, D.; Molofsky, A.; Liang, H.-E.; Ricardo-Gonzalez, R.; Jouihan, H.A.; Bando, J.; Chawla, A.; Locksley, R.M. Eosinophils Sustain Adipose Alternatively Activated Macrophages Associated with Glucose Homeostasis. Science 2011, 332, 243-247. [CrossRef] [PubMed]

54. Calco, G.N.; Fryer, A.D.; Nie, Z. Unraveling the connection between eosinophils and obesity. J. Leukoc. Biol. 2020, 108, 123-128. [CrossRef] [PubMed]

55. Murata, A.; Hyogo, H.; Nonaka, M.; Sumioka, A.; Suehiro, Y.; Furudoi, A.; Fujimoto, Y.; Aisaka, Y.; Komatsu, H.; Tokumo, H. Overlooked muscle cramps in patients with chronic liver disease: In relation to the prevalence of muscle cramps. Eur. $J$. Gastroenterol. Hepatol. 2019, 31, 375-381. [CrossRef]

56. Mehta, S.S.; Fallon, M.B. Muscle Cramps in Liver Disease. Clin. Gastroenterol. Hepatol. 2013, 11, 1385-1391. [CrossRef] 
57. Barbe, M.F.; Elliott, M.B.; Abdelmagid, S.M.; Amin, M.; Popoff, S.N.; Safadi, F.F.; Barr, A.E. Serum and tissue cytokines and chemokines increase with repetitive upper extremity tasks. J. Orthop. Res. 2008, 26, 1320-1326. [CrossRef]

58. Mikova, O.; Yakimova, R.; Bosmans, E.; Kenis, G.; Maes, M. Increased serum tumor necrosis factor alpha concentrations in major depression and multiple sclerosis. Eur. Neuropsychopharmacol. 2001, 11, 203-208. [CrossRef]

59. Strawbridge, R.; Arnone, D.; Danese, A.; Papadopoulos, A.S.; Vives, A.R.H.; Cleare, A.J. Inflammation and clinical response to treatment in depression: A meta-analysis. Eur. Neuropsychopharmacol. 2015, 25, 1532-1543. [CrossRef]

60. Kawaguchi, T.; Taniguchi, E.; Itou, M.; Sumie, S.; Oriishi, T.; Matsuoka, H.; Nagao, Y.; Sata, M. Branched-chain amino acids improve insulin resistance in patients with hepatitis C virus-related liver disease: Report of two cases. Liver Int. 2007, 27, 1287-1292. [CrossRef]

61. Kawaguchi, T.; Nagao, Y.; Matsuoka, H.; Ide, T.; Sata, M. Branched-chain amino acid-enriched supplementation improves insulin resistance in patients with chronic liver disease. Int. J. Mol. Med. 2008, 22, 105-112. [CrossRef]

62. Nagao, Y.; Matsuoka, H.; Kawaguchi, T.; Sata, M. Aminofeel improves the sensitivity to taste in patients with HCV-infected liver disease. Med. Sci. Monit 2010, 16, 7-12.

63. Nagao, Y.; Kawaguchi, T.; Kakuma, T.; Ide, T.; Sata, M. Post-marketing surveillance study for efficacy and safety of Aminofeel, a branched chain amino acids-enriched supplement including zinc. J. New Rem. Clin. 2011, 60, 1046-1063. Available online: https: / / mol.medicalonline.jp / archive/search?jo=aa9syrsc\&vo=60\&nu=5 (accessed on 29 September 2021). (In Japanese).

64. Nagao, Y.; Kawaguchi, T.; Ide, T.; Sata, M. Effect of branched-chain amino acid-enriched nutritional supplementation on interferon therapy in Japanese patients with chronic hepatitis C virus infection: A retrospective study. Virol. J. 2012, 9, 282. [CrossRef]

65. Kawaguchi, T.; Nagao, Y.; Abe, K.; Imazeki, F.; Honda, K.; Yamasaki, K.; Miyanishi, K.; Taniguchi, E.; Kakuma, T.; Kato, J.; et al. Effects of branched-chain amino acids and zinc-enriched nutrients on prognosticators in HCV-infected patients: A multicenter randomized controlled trial. Mol. Med. Rep. 2014, 11, 2159-2166. [CrossRef]

66. Sako, K. Branched-chain amino acids supplements in the late evening decrease the frequency of muscle cramps with advanced hepatic cirrhosis. Hepatol. Res. 2003, 26, 327-329. [CrossRef]

67. Hidaka, H.; Nakazawa, T.; Kutsukake, S.; Yamazaki, Y.; Aoki, I.; Nakano, S.; Asaba, N.; Minamino, T.; Takada, J.; Tanaka, Y.; et al. The efficacy of nocturnal administration of branched-chain amino acid granules to improve quality of life in patients with cirrhosis. J. Gastroenterol. 2012, 48, 269-276. [CrossRef] [PubMed]

68. Raman, M.; Ahmed, I.; Gillevet, P.M.; Probert, C.S.; Ratcliffe, N.M.; Smith, S.; Greenwood, R.; Sikaroodi, M.; Lam, V.; Crotty, P.; et al. Fecal Microbiome and Volatile Organic Compound Metabolome in Obese Humans with Nonalcoholic Fatty Liver Disease. Clin. Gastroenterol. Hepatol. 2013, 11, 868-875. [CrossRef] [PubMed]

69. Goshima, S.; Kanematsu, M.; Kobayashi, T.; Furukawa, T.; Zhang, X.; Fujita, H.; Watanabe, H.; Kondo, H.; Moriyama, N.; Bae, K.T. Staging hepatic fibrosis: Computer-aided analysis of hepatic contours on gadolinium ethoxybenzyl diethylenetriaminepentaacetic acid-enhanced hepatocyte-phase magnetic resonance imaging. Hepatology 2011, 55, 328-329. [CrossRef]

70. Miele, L.; Valenza, V.; La Torre, G.; Montalto, M.; Cammarota, G.; Ricci, R.; Mascianà, R.; Forgione, A.; Gabrieli, M.L.; Perotti, G.; et al. Increased intestinal permeability and tight junction alterations in nonalcoholic fatty liver disease. Hepatology 2009, 49, 1877-1887. [CrossRef] [PubMed] 